\title{
"Efectividad de una intervención educativa en el conocimiento de loncheras saludables y su contenido en las madres de preescolares"
}

"Effectiveness of an educational intervention on knowledge of healthy lunch boxes and their contents in mothers of preschool”

Guisela Quilca Calatayud ${ }^{1}$, Roxana Ramírez Aliaga ${ }^{2}$, Walter Gómez Gonzales ${ }^{3}$

\section{RESUMEN}

Objetivo: Determinar la efectividad de una intervención educativa en el nivel de conocimiento de la preparación de loncheras saludables, en madres de niños preescolares de la Institución Educativa Patricia Teresa Rodríguez 3019 Rímac, 2015.Materiales y métodos: Según el enfoque fue cuantitativo, diseño preexperimental y longitudinal. La muestra estuvo constituida por 25 madres del colegio Patricia Teresa Rodríguez 3019 - UGEL 02 Rímac, el instrumento utilizado fue un cuestionario y se procesaron los datos con el programa SPSS versión 25, para el análisis del efecto de la intervención educativa la prueba t student, aceptándose valores como significativo p < 0.05. Resultados: Antes de la intervención educativa 52\% (13) de madres tuvieron un nivel de conocimiento bajo sobre la lonchera saludable, después de la intervención educativa 100\% (25) obtuvo nivel alto. Antes de la intervención educativa 68\% (17) tuvieron un nivel bajo de conocimientos sobre los tipos de alimentos, después de la intervención educativa el 96\% (24) obtuvo un nivel alto. Antes de la intervención educativa 56\% (14) tuvieron nivel bajo de conocimientos sobre las formas de preparación de loncheras saludables y el 44\% (11) obtuvieron un nivel medio, después de la intervención educativa 100\% (25) obtuvo nivel alto. Antes de la intervención educativa 64\% (16) obtuvo nivel bajo de conocimientos sobre el contenido de las loncheras saludables, después de intervención educativa el 100\% (25) de madres obtuvo un nivel alto. Conclusión: La intervención educativa fue efectiva en los conocimientos de las madres sobre la lonchera saludable, evidenciándose mejoramiento de éstos después de su participación, obteniendo la totalidad de madres un nivel alto de conocimientos.

Palabras Clave: Intervención educativa, conocimientos, loncheras saludables.

\begin{abstract}
Objective: To determine the effectiveness of an educational intervention on the level of knowledge and practice of preparing healthy lunchboxes, mothers of preschool children of School Patricia Teresa Rodriguez 3019 Rimac, 2015.Materials and Methods: According to the approach was quantitative, pre-experimental and longitudinal design. The sample consisted of 25 mothers of school Patricia Teresa Rodriguez 3019 UGELs 02 Rímac, the instrument used was a questionnaire and the data were processed with SPSS version 25 for the analysis of the effect of educational intervention t student test, accepting values as significant $\mathrm{p}$ $<$ 0.05.Results: Before educational intervention 52\% (13) of mothers had a low level of knowledge about healthy lunchbox, after the educational intervention 100\% (25) achieved high level. Before the educational intervention 68\% (17) had a low level of knowledge about the types of food, after the educational intervention $96 \%$ (24) achieved a high level. Before the educational intervention 56\% (14) had low level of knowledge about ways of preparing healthy lunchboxes and 44\% (11) had an average level after the educational intervention 100\% (25) achieved high level. Before the educational intervention 64\% (16) scored low level of knowledge about the content of healthy lunchboxes, after educational intervention $100 \%$ (25) of mothers he received a high level. Conclusion: The educational intervention was effective in the knowledge of mothers about healthy lunchbox, showing their improvement after their participation, obtaining all mothers a high level of knowledge.
\end{abstract}

Key words: Educational intervention, knowledge, healthy lunchboxes.

${ }^{1}$ Licenciada en Enfermería. Universidad Norbert Wiener.

${ }^{2}$ Licenciada en Enfermería. Universidad Norbert Wiener.

${ }^{3}$ Doctor Ciencias de la Salud. Universidad Norbert Wiener. 


\section{INTRODUCCION}

El desarrollo humano es considerado el eje fundamental en el desarrollo de las naciones; cada día surgen más investigaciones que demuestran que la adecuada nutrición en los diferentes ciclos de vida del individuo constituye uno de los factores básicos de este.

La alimentación durante el periodo de gestación, lactancia así como los primeros años de vida del ser humano resulta esencial para posibilitar el óptimo desarrollo de las potencialidades del individuo, las mismas que son indispensables para el mejoramiento de la productividad, crecimiento económico y desarrollo social sostenido (1).

Uno de los principales problemas que aqueja al Perú y al mundo en la actualidad, es la malnutrición infantil, producida por el consumo inadecuado en cantidad y calidad de los alimentos, utilizándose por lo tanto a nivel corporal menor o mayor cantidad de energía, modificando su funcionamiento y generando alteraciones nutricionales tanto por defecto como por exceso.

La etapa preescolar es un período muy importante donde se debe cuidar su nutrición, alimentación y requerimientos de energía; el consumo de proteínas, vitaminas y minerales son diferentes a los establecidos para los lactantes, escolares y adultos. Se requiere que se establezcan preferencias alimentarias individuales, tanto del desayuno como el contenido de la lonchera.

Existe un elevado porcentaje de malnutrición en los niños pequeños, que tiene graves repercusiones en su desarrollo, pues esos primeros años de vida son fundamentales para su crecimiento y desarrollo. Las aptitudes y actitudes básicas se forjan en la primera infancia; además, en ese periodo del desarrollo hay una gran demanda de energía para satisfacer las necesidades biológicas y sociales de crecimiento y maduración. Los niños que sufren de malnutrición pertenecen a las familias con niveles más bajos de ingresos y de educación dentro de la comunidad. Este contexto ambiental puede acentuar los efectos perjudiciales de las carencias nutricionales y viceversa (2).

La Organización Mundial de la Salud (2015) indica que dentro de los casos de malnutrición se encuentra la desnutrición que a nivel mundial hay cerca de 115 millones de niños con insuficiencia ponderal; (peso por debajo de los parámetros normales), la desnutrición contribuye a cerca de un tercio de la mortalidad infantil; el retraso del crecimiento (un indicador de desnutrición crónica) dificulta el desarrollo de 171 millones de niños menores de cinco años, más de una tercera parte de los niños en edad preescolar del mundo presenta deficiencia de vitamina A y hierro; la desnutrición del niño suponen más del $10 \%$ de la carga de morbilidad mundial (3).

Según el Fondo de Naciones Unidas para la Infancia (UNICEF) en el informe "Mejorando la nutrición infantil” en Latinoamérica y el Caribe el porcentaje de niños menores desnutridos de 5 años se redujo de $22 \%$ en 1990 a $12 \%$ en 2012, además, destaca el caso peruano que con políticas intersectoriales y consistentes desde la Iniciativa de la Desnutrición Infantil el 2006 y la Estrategia Nacional para la Reducción de la Pobreza y Oportunidades Económicas (CRECER) el 2007, de $30 \%$ entre 2004 y 2006, se redujo a 20\% en 2011. Sin embargo, advierte que por desnutrición crónica infantil hay más de medio millón de niños peruanos menores de 5 años con retraso de crecimiento. Dentro de los 9 países de Latinoamérica y el Caribe involucrados en el informe, solo Colombia registra mayor número de niños en esta condición (595,000), y después de Perú $(566,000)$, Venezuela (458,000), Bolivia (333,000), Nicaragua (149,000), El Salvador (121,000), República Dominicana $(103,000)$, Panamá $(66,000)$ y Costa Rica (20,000).

Según el Instituto Nacional de Estadística e Informática (INEI), el porcentaje de desnutrición crónica infantil a nivel nacional es $19.5 \%$ al 2012 y $18.1 \%$ al 2013, sin embargo, estas cifras globales no expresan la realidad de muchas regiones y zonas rurales pobres con cifras altísimas, por ejemplo, el 82\% de niños ashánincas sufren desnutrición crónica y en la región Loreto 33\%; las regiones mineras lideran las tasas: Huancavelica (52\%), Cajamarca (36\%), Apurímac (31\%) y Ayacucho (28\%). También en el ámbito rural se registra 37\%, más del triple del urbano (10.1\%), y en la Sierra global 35\%. En Lima metropolitana el 6.8\% de niños están desnutridos y 111,000 niños menores de 3 años padecen de anemia.

En el Perú, dentro de la cultura nutricional alimenticia familiar, el consumo de alimentos tradicionales ha disminuido considerablemente, incrementándose la frecuencia del consumo de alimentos con poco valor nutritivo o "alimentos chatarra”; y dado que las costumbres y hábitos alimenticios de los padres determinan la alimentación del niño, éste inicia desde temprana edad el consumo de dichos alimentos por el menor costo y accesibilidad a éstos, reforzada por los avisos publicitarios, promoviendo por lo tanto una alimentación infantil deficiente. 
Uno de los complementos del desayuno del preescolar es la lonchera, que debe contener alimentos que contribuye a que el niño mantenga una dieta balanceada y realmente nutritiva. Sus funciones son: mantener suficientes reservas de nutrientes durante el día escolar, suministrar alimentos nutritivos que al pequeño le gustan y crear hábitos saludables de alimentación.

Especialistas de la Dirección de Educación para la Salud del Ministerio de Salud (MINSA), explicaron que el refrigerio escolar saludable permite a sus hijos asegurar el aporte de energía y nutrientes que necesitan durante el tiempo que permanecen en la escuela. Este debe ser fácil de preparar, práctico de llevar, ligero, nutritivo, económico y sobretodo, sabroso. Debe prepararse con alimentos variados, naturales y de alto valor nutricional, como las frutas y verduras, cereales integrales como avena, maíz cancha, mote, trigo, quinua, kiwicha, cañihua; menestras (4).

El proporcionar a los niños una dieta balanceada y saludable es parte del rol cuidador ejercido por las madres y/o cuidadores; evidenciándose a través del conocimiento y selección de alimentos nutritivos en la alimentación diaria del niño, entre ellos la lonchera, la que permitirá cubrir sus requerimientos energéticos para un adecuado rendimiento físico y mental en sus actividades escolares.

Es necesaria una iniciativa concientizadora educativa en las madres y es función de los profesionales de enfermería, como parte del ejercicio del cuidado holístico, contribuyendo al bienestar de los niños, creando condiciones individuales, familiares y sociales que le permitan desenvolverse acertadamente $\mathrm{y}$ asegurando finalmente un adecuado crecimiento y desarrollo biposicosocial.

Dentro de las actividades preventivas promocionales del Centro de Salud Villa Los Ángeles, y en coordinación con la Institución Educativa Patricia Teresa Rodríguez, al realizar la evaluación de peso y talla en los niños se pudo evidenciar a niños con bajo peso, otros con sobrepeso y talla inadecuada para su edad. Al verificar el contenido de las loncheras, se encontró alimentos no adecuados nutricionalmente para el niño, es decir, "alimentos chatarra" tales como dulces, galletas, refrescos en cajas que contienen mucha azúcar, embutidos y gaseosas.

Al interrogar a algunas madres de estos niños preescolares, refieren "que estos alimentos enlatados son más higiénicos y fáciles de preparar y que se conservan mejor, que no tienen tiempo para cocinar y que a los niños les gusta más los frugos y gaseosas", entre otros comentarios. El objetivo del estudio fue, determinar la efectividad de una intervención educativa en el conocimiento de loncheras saludables y su contenido, en madres de niños preescolares de la Institución Educativa Patricia Teresa Rodríguez 3019 Rímac, 2015.

\section{MATERIALES Y METODOS}

Tipo y método de Investigación: El presente estudio fue de tipo cuantitativo ya que es susceptible de cuantificación, nivel aplicativo ya que busca mejorar los conocimientos y prácticas sobre la preparación de loncheras que realizan las madres de los preescolares, es de método pre experimental porque nos va a permitir obtener información basada en la realidad y poder aplicar intervenciones en cuanto a los resultados encontrados, en un determinado tiempo y espacio. Longitudinal porque se recolectarán los datos en diferentes oportunidades.

Población y Muestra: La población estuvo conformada por 99 madres de los niños de 3 a 5 años de edad, que asisten para recoger a sus niños que estudian en el turno mañana del nivel inicial del colegio Patricia Teresa Rodríguez 3019 UGEL 02 Rímac. La muestra fue de 25 madres de familia que asistieron a las 3 sesiones educativas y se les aplicó el pre y post test. Fue una muestra no probabilística a conveniencia y se tuvo en cuenta los siguientes criterios:

\section{Criterios de Inclusión:}

- Madres que acudieron a recoger a sus niños de 3 a 5 años de edad matriculados y que asisten regularmente a la institución Educativa.

- Madres que acepten participar en el trabajo de investigación.

\section{Criterios de Exclusión:}

- Madres que no acepten participar de la Intervención Educativa y trabajo de investigación. - Madres que no hable castellano.

\section{Técnicas e Instrumentos de recolección de datos}

La técnica que se utilizó fue la encuesta y el instrumento un formulario tipo cuestionario, el cual consta de las siguientes partes: Introducción, datos generales de la madre, conocimientos que tienen las madres en la preparación de las loncheras con 10 preguntas de opción múltiple y prácticas

Ágora Rev Cient.2015; 03(01):320-328 
sobre el contenido de las loncheras con 10 preguntas de opción múltiple.

El cuestionario utilizado en el estudio fue validado por juicio de expertos que dio un grado de concordancia significativo de 0.04 . La validez se determinó por medio de la correlación de $\mathrm{R}$ de Pearson obteniendo un puntaje de 0.665 y la confiabilidad por Kuder - Richarson se obtuvo 0.71 donde se concluye que el instrumento es altamente confiable.

La categorización de los conocimientos y prácticas fue de la siguiente manera:

- $\quad$ Nivel Alto: De 15 a 20 puntos

- $\quad$ Nivel Medio: De 10 a 14 puntos

- $\quad$ Nivel Bajo: De 0 a 9 puntos

Para la realización del presente trabajo de investigación se coordinó con las autoridades del Centro Educativo Patricia Teresa Rodríguez 3019 - RIMAC, mediante un documento emitido por la universidad. Se coordinó con la Directora y docentes encargadas de los preescolares para la aplicación del instrumento a las madres de los preescolares, este medía el conocimiento y prácticas de loncheras saludables. Se estableció el cronograma de sesiones educativas con técnica Expositiva- Participativa, los cuales se llevaron a cabo en tres días, 1 por semana, 2 sesiones teóricas: Lonchera Saludable y Contenido de lonchera saludable, y una sesión demostrativa sobre la preparación de la lonchera saludable.
Previamente se aplicó el consentimiento informado a las madres después de explicarles en qué consistiría el trabajo de investigación y la importancia de su participación. Luego se realizó la recolección de datos, aplicándose el instrumento tipo cuestionario a las madres, para lo cual se les dio un tiempo promedio de 20 minutos para desarrollarlo (pre test). Después de concluidas las sesiones teóricas y prácticas se aplicó nuevamente el cuestionario para evaluar los resultados de la Intervención educativa (post test).

\section{Procesamiento de datos y análisis estadístico}

El procesamiento de datos se realizó con el programa estadístico SPSS, versión 25 para Windows en español y para el análisis del efecto de la intervención educativa la prueba t student, una de las pruebas más apropiadas cuando dos muestras independientes de observaciones se miden en una escala de intervalo, aceptándose valores como significativo $\mathrm{p}<0.05$.

\section{Aspectos Éticos}

Para la realización del presente estudio se realizaron las gestiones administrativas correspondientes con las autoridades pertinentes de dicha institución para contar con la autorización respectiva. La recolección de los datos se realizó con las madres de los preescolares de dicha institución educativa que desearon participar de manera voluntaria, teniendo la opción de retirarse al momento que lo crean pertinente; respetando así su autonomía.

\section{RESULTADOS}

En la Tabla 1, se evidencia que antes de la intervención educativa el 52\% (13) de madres tuvieron un nivel de conocimiento bajo sobre la lonchera saludable, el $48 \%$ (12) nivel medio y ninguna de las madres tuvo un nivel de conocimiento alto.

Tabla 1: Nivel de conocimiento sobre loncheras saludables antes de la intervención educativa en madres de preescolares de la IE “Patricia Teresa Rodríguez 3019” SMP. 2015

\begin{tabular}{ccc}
\hline \hline \multirow{2}{*}{ Nivel de conocimiento } & \multicolumn{2}{c}{ Antes de IE } \\
& $\mathrm{f}$ & $\%$ \\
\hline Alto & 0 & $0 \%$ \\
Medio & 12 & $48 \%$ \\
Bajo & 13 & $52 \%$ \\
TOTAL & $\mathbf{2 5}$ & $\mathbf{1 0 0 \%}$ \\
\hline
\end{tabular}

En la Tabla 2, se evidencia que después de la intervención educativa el 100\% (25) de las madres obtuvo un nivel de conocimiento alto sobre loncheras saludables. 
Tabla 2: Nivel de conocimiento sobre loncheras saludables después de la intervención educativa en madres de preescolares de la IE "Patricia Teresa Rodríguez 3019” SMP. 2015

\begin{tabular}{ccc}
\hline \hline \multirow{2}{*}{ Nivel de conocimiento } & \multicolumn{2}{c}{ Después de la IE } \\
& $\mathrm{f}$ & $\%$ \\
\hline \hline Alto & 25 & $100 \%$ \\
Medio & 0 & 0 \\
Bajo & 0 & 0 \\
TOTAL & 25 & $100 \%$ \\
\hline
\end{tabular}

En la Tabla 3, se evidencia que después de la intervención educativa la totalidad de madres, es decir 100\% (25) logró un nivel de conocimiento alto con respecto a las loncheras saludable.

Tabla 3: Nivel de conocimiento sobre loncheras saludables antes y después de la intervención educativa en madres de preescolares de la IE “Patricia Teresa Rodríguez 3019” SMP. 2015

\begin{tabular}{ccccc}
\hline \multirow{2}{*}{ Nivel de conocimiento } & \multicolumn{3}{c}{ Antes de IE } & \multicolumn{2}{c}{ Después de la } \\
& f & $\%$ & f & $\%$ \\
\hline Alto & 0 & $0 \%$ & 25 & $100 \%$ \\
Medio & 12 & $48 \%$ & 0 & 0 \\
Bajo & 13 & $52 \%$ & 0 & 0 \\
TOTAL & $\mathbf{2 5}$ & $\mathbf{1 0 0 \%}$ & 25 & $100 \%$ \\
\hline
\end{tabular}

En la Tabla 4, se evidencia que antes de la intervención educativa el 68\% (17) de madres tuvieron un nivel de conocimiento bajo sobre los tipos de alimentos y el 32\% (8) nivel medio. Después de la intervención educativa el 96\% (24) de madres obtuvo un nivel alto y sólo el 4\% (1) obtuvo un nivel medio.

Tabla 4: Nivel de conocimiento sobre tipos de alimentos antes y después de la intervención educativa en madres de preescolares de la IE “Patricia Teresa Rodríguez 3019” SMP. 2015

\begin{tabular}{ccccc}
\hline \multirow{2}{*}{ TIPOS DE ALIMENTOS } & \multicolumn{2}{c}{ Antes } & \multicolumn{2}{c}{ Después } \\
& $\mathrm{f}$ & $\%$ & $\mathrm{~F}$ & $\%$ \\
\hline \hline Alto & 0 & $0 \%$ & 24 & $96 \%$ \\
Medio & 8 & $32 \%$ & 1 & $4 \%$ \\
Bajo & 17 & $68 \%$ & 0 & $0 \%$ \\
TOTAL & 25 & $100 \%$ & 25 & $100 \%$ \\
\hline
\end{tabular}

En la Tabla 5, se evidencia que antes de la intervención educativa el 56\% (14) de madres tuvieron un nivel de conocimiento bajo sobre las formas de preparación de las loncheras saludables y el $44 \%$ (11) obtuvieron un nivel medio. Después de la intervención educativa el 100\% (25) de madres obtuvo un nivel alto.

Tabla 5: Nivel de conocimiento sobre formas de preparación de las loncheras saludables antes y después de la intervención educativa en madres de preescolares de la IE "Patricia Teresa Rodríguez 3019” SMP. 2015

\begin{tabular}{ccccc}
\hline \hline FORMA DE & \multicolumn{2}{c}{ Antes } & \multicolumn{2}{c}{ Después } \\
PREPARACIÓN & $\mathrm{f}$ & $\%$ & $\mathrm{~F}$ & $\%$ \\
\hline \hline Alto & 0 & $0 \%$ & 25 & $100 \%$ \\
Medio & 11 & $44 \%$ & 0 & 0 \\
Bajo & 14 & $56 \%$ & 0 & 0 \\
TOTAL & 25 & $100 \%$ & 25 & $100 \%$ \\
\hline
\end{tabular}

En la Tabla 6, se evidencia que antes de la intervención educativa el 64\% (16) de madres tuvieron un nivel de conocimiento bajo sobre el contenido de las loncheras saludables y el 36\% (9) obtuvieron un nivel medio. Después de la intervención educativa el 100\% (25) de madres obtuvo un nivel alto. 
Tabla 6: Nivel de conocimiento sobre contenido de la lonchera saludable antes y después de la intervención educativa en madres de preescolares de la IE “Patricia Teresa Rodríguez 3019” SMP. 2015

\begin{tabular}{ccccc}
\hline \hline CONTENIDO DE LA & \multicolumn{2}{c}{ Antes } & \multicolumn{2}{c}{ Después } \\
LONCHERA & f & $\mathbf{\%}$ & $\mathbf{f}$ & $\mathbf{\%}$ \\
SALUDABLE & & 0 & $\mathbf{2 5}$ & $100 \%$ \\
\hline \hline Alto & $\mathbf{0}$ & $0 \%$ & $\mathbf{0}$ & 0 \\
Medio & $\mathbf{9}$ & $36 \%$ & $\mathbf{0}$ & 0 \\
Bajo & $\mathbf{1 6}$ & $64 \%$ & $\mathbf{0}$ & $100 \%$ \\
TOTAL & $\mathbf{2 5}$ & $100 \%$ & $\mathbf{2 5}$ & \\
\hline
\end{tabular}

La variación del promedio durante el proceso de la intervención respecto al pretest, se evaluó aplicando la prueba "t" de student para grupos homogéneos, los resultados muestran que la intervención educativa efectuada fue efectiva en el cambio sobre el nivel de conocimientos de las madres sobre el contenido de las loncheras saludables; obteniéndose una diferencia altamente significativa en la prueba de hipótesis $t=4,243$ $\mathrm{p}<0,01$, respecto al control pretest.

\section{Puntajes totales de conocimientos, obtenidos por las madres antes y después de la intervención} educativa

\begin{tabular}{|c|c|c|}
\hline Madres & Nivel de conocimiento antes de la IE & Nivel de conocimiento después de la IE \\
\hline 1 & 4 & 15 \\
\hline 2 & 5 & 16 \\
\hline 3 & 6 & 15 \\
\hline 4 & 6 & 18 \\
\hline 5 & 6 & 16 \\
\hline 6 & 7 & 17 \\
\hline 7 & 7 & 18 \\
\hline 8 & 7 & 18 \\
\hline 9 & 7 & 19 \\
\hline 10 & 8 & 16 \\
\hline 11 & 8 & 17 \\
\hline 12 & 9 & 19 \\
\hline 13 & 10 & 16 \\
\hline 14 & 10 & 18 \\
\hline 15 & 10 & 19 \\
\hline 16 & 10 & 18 \\
\hline 17 & 10 & 19 \\
\hline 18 & 11 & 20 \\
\hline 19 & 11 & 17 \\
\hline 20 & 11 & 16 \\
\hline 21 & 12 & 17 \\
\hline 22 & 12 & 18 \\
\hline 23 & 12 & 20 \\
\hline 24 & 13 & 19 \\
\hline 25 & 14 & 20 \\
\hline $\mathrm{X}=$ Media aritmética & $\mathrm{X} 1=9.04$ & $\mathrm{X} 2=17.64$ \\
\hline
\end{tabular}




\section{DISCUSIÓN}

Con respecto a las características sociodemográficas de las madres de los preescolares se encontró que el $40 \%$ (25) tiene entre 23 a 28 años de edad y el 8\% (2) tiene entre 43 a 50 años; el 36\% (9) ha estudiado secundaria completa y la misma cantidad tiene secundaria incompleta, el 20\% (5) tiene estudios técnico o superior. El 76\% (19) son de Lima y el 24\% (6) son de la Sierra y Selva; el 36\% (9) tienen 2 hijos y el $16 \%$ (4) tiene entre 4 a más hijos. El 72\% (18) de las madres son amas de casa que se dedican a su hogar. En el estudio se halló, que antes de la intervención educativa el 52\% (13) de madres tuvieron un nivel de conocimiento bajo sobre la lonchera saludable y el 48\% (12) nivel medio y después de la intervención educativa el 100\% (25) de las madres obtuvo un nivel de conocimiento y práctica alto.

Similares resultados encontró Bonilla Arce, Silvia, Perú (5), en su investigación sobre el efecto de intervención educativa sobre el nivel de conocimiento de las madres y mejora de preparación de lonchera del preescolar: el 50\% (15) tienen un nivel de conocimiento medio, el 23\% (7) alto y $27 \%$ (8) bajo sobre la preparación de la lonchera saludable antes de la intervención y después de recibir la intervención educativa, el $100 \%$ (30) alcanzó un nivel de conocimientos alto. Las loncheras permiten complementar las necesidades nutricionales del niño durante el día permitiéndole reponer la energía gastada durante las actividades diarias, y mejorar su concentración durante el estudio. En la actualidad existe marcado desconocimiento en nutrición infantil y su relación con el crecimiento y desarrollo de los niños, evidenciado por el consumo frecuente de alimentos chatarra en las loncheras del preescolar, creando inadecuados hábitos alimenticios que repercutirán en su salud presente y futura. Las madres u otro familiar quienes asumen el rol de cuidador del preescolar, deben estar informadas y preparadas para ejercer adecuadamente el "cuidado" que asegure la protección y preservación de la salud del niño, enfatizando en el aspecto nutricional para garantizar un óptimo crecimiento y desarrollo infantil, a través del conocimiento y selección de alimentos nutritivos en la alimentación diaria del niño, entre ellos la lonchera. La educación en salud es una estrategia fundamental que permiten a las personas incrementar el control sobre su salud, desarrollar a la par habilidades personales y actitudes saludables para prevenir enfermedades y crear un futuro más saludable. El nivel de conocimiento del individuo está directamente relacionado con el alcance a la información que se tiene sobre determinado tema y a su vez influenciado por la edad y el grado de instrucción. Un estudio realizado por el Instituto Nacional de Estadística concuerda con lo mencionado al demostrar que a menor grado de instrucción, es mayor el porcentaje de niños con deficiente estado nutricional, debido a la falta de acceso a la educación formal. Por otro lado, las madres con mayor nivel educativo tienen mejor manejo de información, por lo que se asignan de forma más eficiente los recursos dentro del hogar (6)

Con respecto a los objetivos específicos: Identificar los conocimientos y prácticas en la preparación de loncheras saludables: tipos de alimentos, formas de preparación y contenido, en las madres de preescolares antes y después de la aplicación de la intervención educativa (pre test post test).

En la presente investigación, los conocimientos y prácticas sobre los tipos de alimentos se encontró que antes de la intervención educativa el 68\% (17) de madres tuvieron un nivel de conocimiento y prácticas bajo y el 32\% (8) un nivel medio. Después de la intervención educativa el 96\% (24) de las madres obtuvo un nivel alto y sólo el 4\% (1) medio. Resultados similares encontró Peña H. Dean, Perú (7), en su investigación titulada efectividad de un programa educativo en el incremento de conocimientos en las madres de niños en edad preescolar sobre la preparación de loncheras saludables, en que el 61\% (17) cuidadores desconocen sobre la inclusión de las proteínas dentro de la lonchera saludable. Así mismo el 61\% (17) madres desconocen sobre la combinación de alimentos energéticos, constructores y reguladores dentro de la lonchera preescolar. El 61\% (17) madres desconocen sobre el queso como fuente importante de calcio. La lonchera debe contener alimentos energéticos, reguladores y constructores. Sus funciones son mantener suficientes reservas de nutrientes durante el día escolar, suministrar alimentos nutritivos que al pequeño le gusten y crear hábitos saludables de alimentación. Es importante conjugar estratégicamente los componentes nutricionales como los alimentos energéticos, constructores y reguladores para que sea una lonchera saludable. $\mathrm{Y}$ para ello es necesario que la madre tenga los conocimientos claros y precisos de cada elemento para preparar loncheras nutritivas; y es la enfermera, el profesional de salud que trabaja en el área público-privado que debe incluir en su plan de intervención comunitaria brindar educación oportuna a las madres de familia utilizando técnicas educativas interactivas y participativas 
que contribuyan a la adquisición de conocimientos para la alimentación infantil.

En el estudio se encontró que antes de la intervención educativa el 56\% (14) de madres tuvieron un nivel de conocimiento bajo sobre las formas de preparación de las loncheras saludables y el $44 \%$ (11) obtuvieron un nivel medio. Después de la intervención educativa el $100 \%$ (25) de madres obtuvo un nivel alto. Con respecto al contenido de la lonchera se encontró que antes de la intervención educativa el 64\% (16) de madres tuvieron un nivel de conocimiento bajo y el 36\% (9) obtuvieron un nivel medio. Después de la intervención educativa el 100\% (25) de madres obtuvo un nivel alto.

Similares resultados encontró Infante C, María, Perú (8) en su investigación titulada: Efectividad de una intervención educativa en el mejoramiento de los conocimientos sobre la preparación de la lonchera, que tienen las madres de preescolares, antes de la intervención educativa se encontró que $44 \%$ (11) no conocen ni practican la forma adecuada de preparación de la lonchera, el 62\% (16) no conocen ni practican la frecuencia de alimentos saludables en la lonchera, el 68\% (18) no conocen ni practican la cantidad necesaria en bebidas no colorantes. Después de aplicar la intervención educativa se encontró que el 80\% (22) conocen y practican la forma adecuada de preparación de la lonchera, 74\% (18) conocen y practican la frecuencia de alimentos saludables en la lonchera.

La buena alimentación del niño en edad pre escolar provee la suficiente energía física y mental para enfrentar adecuadamente las actividades del día. Un niño mal alimentado presentará problemas e irreversiblemente dificultades para concentrarse y finalmente aprender, por ello es muy importante que los niños tomen un buen desayuno y lleve una lonchera nutritiva. Esta debe contener alimentos energéticos, reguladores y constructores, que se traduce en lo siguiente: un alimento sólido (proteína), una fruta y líquidos (refresco). Este complemento alimenticio es sumamente importante ya que los niños como están en actividad constante necesitan energía varias veces al día (en pequeñas porciones; porque la capacidad gástrica del niño es mucho menor que la de los adultos). La lonchera preescolar nutricionalmente ideal se encuentra compuesta por distintos grupos de alimentos, variados y balanceados, capaces de cubrir los requerimientos del preescolar, siendo la lonchera un complemento alimenticio, indispensable para lograr un óptimo desarrollo en nuestros niños, es una de las cinco comidas necesarias para un niño, ya que las tres comidas fuertes (desayuno, almuerzo y comida) y dos meriendas (a media mañana y a media tarde) son indispensables para el niño. Ninguna influencia es mayor sobre los hábitos alimenticios que las que existe dentro del hogar, el aprendizaje de hábitos alimenticios a través de vida, así de conocimientos y de información determina comportamientos equivocados y erróneos con respecto a la salud y enfermedad, situación que debe ser tomada en cuenta sobre todo en la madre por ser sin duda el eje de la función familiar, la depositaria del direccionamiento en la salud del niño y demás miembros de la familia.

\section{CONCLUSION}

La intervención educativa fue efectiva en los conocimientos de las madres sobre la lonchera saludable, evidenciándose mejoramiento de éstos después de su participación en la misma, obteniendo la totalidad de madres un nivel alto de conocimientos.

\section{AUTOR DE CORRESPONDENCIA}

Walter Edgar Gómez Gonzales

Universidad Norbert Wiener - UNW.

Avenida Arequipa 440-Santa Beatriz-Lima

Teléfono: 01-706-5100

E-mail: waltergomez29@yahoo.com

\section{REFERENCIAS BIBLOGRAFICAS}

1. Ministerio de Salud del Perú. Lineamientos de política Sectorial para el periodo 2002 - 2012 Lima 2012 (citado 25 de marzo 2014). Disponible en http://www.lib.utexas.edu/benson/lagovd ocs/peru/federal/salud/planestrategico/pol itica-sectorial-2002-2012.pdf.

2. Serie de Educación sobre Nutrición UNESCO ORGANIZACIÓN DE LAS NACIONES UNIDAS / Ernesto Pollitt 2015 - Paris disponible en: http://unesdoc.unesco.org/images/0006/0 00623/062306so.pdf

3. Organización Mundial de la Salud/Programas y Proyectos Desafíos Nutrición - 2015 disponible en: http://www.who.int/nutrition/challenges/e s/

4. Rivera M. Priego H. Córdova J. Programa educativo de alimentación y nutrición en una comunidad del Estado de Tabasco, (Optar Licenciatura) Universidad de Tabasco - México; 2010. 
5. Bonilla Arce, S. Efecto de intervención educativa sobre el nivel de conocimiento de las madres y mejora de preparación de lonchera del preescolar en la I.E.I. 638, San Juan de Miraflores (Optar Licenciatura). Lima - Perú; 2014

6. Ministerio de Salud: Gestión Medica Actualidad / 2007 Disponible en: ftp://ftp2.minsa.gob.pe//descargas/ogc/es peciales/2007/nutricion/recortes/12-11-07 nutricion_lav_manos_\%28gm\%29.pdf

7. Peña Huamán, Dean E. Efectividad de un programa educativo en el incremento de conocimientos en las madres de niños en edad preescolar sobre la preparación de loncheras saludables en la institución educativa inicial; "San Martin de Porres" (Optar Licenciatura) Lima - Perú; 2013.
8. Infante C, María. Efectividad de una intervención educativa en el mejoramiento de los conocimientos y prácticas sobre la preparación de la lonchera, que tienen las madres de preescolares en una Institución Educativa Privada en Santa Anita (Optar Licenciatura). Lima - Perú; 2015.

Recibido: 04 /03/2016

Aceptado: 03/06/2016 